\title{
Retinoblastoma cTX TNM Finding v7
}

National Cancer Institute

\section{Source}

National Cancer Institute. Retinoblastoma CTXTNM Finding v7. NCI Thesaurus. Code C88752.

Retinoblastoma in which the primary tumor cannot be assessed. (from AJCC 7th Ed.) 